organ involvement (MS-RO), and 4 multisystem disease with risk organ involvement (MS-RO). Chemotherapy based on vinblastine with corticosteroids was used in 4 patients who had MS-RO form. The outcome was favourable in 6 cases.

Conclusions Langerhans cell histiocytosis is a rare and heterogeneous disease. Multisystem disease with risk organ involvement justify the use of many drugs.

\section{PO-0167 STUDY ON THE FREQUENCY AND CAUSES OF SEVERE IRON DEFICIENCY ANEMIA IN INFANTS AND YOUNG CHILDREN}

${ }^{1} \mathrm{C}$ Singer, ${ }^{1} \mathrm{P}$ Stancu, ${ }^{1} \mathrm{~A}$ Morosanu, ${ }^{2} \mathrm{C}$ Cristea, ${ }^{2} \mathrm{~B}$ Dumitru. ${ }^{1}$ IInd Pediatric Clinic, University of Medicine and Pharmacy of Craiova, Craiova, Romania; ${ }^{2}$ Ind Pediatric Clinic, Emergency Clinical County Hospital Craiova, Craiova, Romania

10.1136/archdischild-2014-307384.830

Objectives To study the frequency and causes of the severe iron deficiency anaemia (AF) (haemoglobin $<7 \mathrm{~g} \%$ ) in infants and young children.

Material and methods We studied the observation sheets of infants and children between 1-3 years hospitalised with AF at the 2nd Paediatric Clinic, EUCH Craiova in the interval 1.01.2011-31.12.2013.

Results AF was recorded in 678 infants and 784 children, with the age between 1-3 years. Severe forms were present in 14 infants and 28 children, age1-3 years. Mean haemoglobin: infant $5.61 \pm 0.79(4,8-7) \mathrm{g} \%$; children $1-3$ years $5.45 \pm 1.2$ (3-7) $\mathrm{g} \%$. Gender distribution of AF severe forms: infant $\mathrm{M} / \mathrm{F}$ : 10/4; children 1-3 years: 18/10; the backgrounds Urban/Rural: infants $3 / 11$; children $1-3$ years $6 / 22$. Severe AF causes in infants: prematurity in 8 cases, prematurity + twins 2 cases, 3 cases with food causes, cystic fibrosis in 1 case. The causes in children with the age between 1-3 years were: food (flour + excess cow's milk) in 23 cases, food intake deficiency in: congenital heart malformations, childhood chronic encephalopathy, palatoschizis /cleft palate, Toxocara canis and parasitic infestation with uncorrected anaemia in infants born prematurely, for each situation 1 case.

Conclusions

1. Severe forms of AF frequency were $2 \%$ in infants with AF and $3.6 \%$ in children with the age between $1-3$ years.

2. Rural origin was over three times higher in both age groups.

3. $2 / 3$ of the infants with severe AF were premature/ twin; food mistakes were the AF cause in $82.1 \%$ of the children aged 1-3 years.

\section{PO-0168 LEUKAEMIA CUTIS: AN UNUSUAL PAEDIATRIC PRESENTATION OF ACUTE LYMPHOBLASTIC LEUKAEMIA}

${ }^{1}$ E Thomas, ${ }^{1} \mathrm{M}$ Young, ${ }^{1} \mathrm{M}$ Wimalendra, ${ }^{2} \mathrm{O}$ Tunstall. ${ }^{1}$ Paediatrics, Plymouth Hospitals NHS Trust, Plymouth, UK; ${ }^{2}$ Department of Paediatric Haematology, University Hospitals Bristol NHS Foundation Trust, Bristol, UK

\subsection{6/archdischild-2014-307384.831}

We present the case of a 12-month-old boy presenting in February 2014 with widespread soft tissue nodules that had progressed over 5 months. They were not painful or itchy and there were no associated symptoms. He had continued to gain weight and had remained systemically well.
He had widespread subcutaneous and firm nodules over his scalp, forehead, trunk, back, abdominal wall and scrotum. They were non-tender and had no overlying skin changes. He was pale but systemic examination was otherwise normal. There was no significant lymphadenopathy or hepatosplenomegaly.

Blood tests confirmed normocytic, hypochromic anaemia $(\mathrm{Hb}$ $69 \mathrm{~g} / \mathrm{L})$, slightly low white cell count $\left(5.5 \times 10^{9} / \mathrm{L}\right)$ and normal platelet count $\left(198 \times 10^{9} / \mathrm{L}\right)$. Over the following week the blood count deteriorated with progressive anaemia and leucopenia with an increasing blast cell population. Tissue biopsy and bone marrow aspirate confirmed a diagnosis of pre-B cell acute lymphoblastic leukaemia (ALL) with mixed-lineage leukaemia (MLL) gene rearrangement.

Cutaneous leukaemia (leukaemia cutis) is a rare presentation of ALL signifying neoplastic infiltration of the skin. The appearance of skin lesions is variable and can manifest in different leukaemia subtypes (most commonly seen in acute myeloid leukaemia and in neonates). Occasionally it may be the only clinical sign of leukaemia but is invariably felt to be a poor prognostic sign.

This case describes an unusual presentation of childhood leukaemia, highlighting the importance of early skin biopsy in unusual cutaneous lesions. To our knowledge it is the first case of cutaneous leukaemia in a child with pre-B ALL with an MLL gene rearrangement.

\section{P0-0169 AN UNUSUAL CASE OF PAINFUL PURPURA - GARDNER-DIAMOND SYNDROME}

${ }^{1}$ E Thomas, ${ }^{1} \mathrm{M}$ Wimalendra, ${ }^{1} \mathrm{~V}$ Ohlsson, ${ }^{2} \mathrm{~J}$ Clinch, ${ }^{3} \mathrm{O}$ Tunstall. ${ }^{1}$ Paediatrics, Plymouth Hospitals NHS Trust, Plymouth, UK; ${ }^{2}$ Department of Paediatric Rheumatology, University Hospitals Bristol NHS Foundation Trust, Bristol, UK; ${ }^{3}$ Department of Paediatric Haematology, University Hospitals Bristol NHS Foundation Trust, Bristol, UK

\subsection{6/archdischild-2014-307384.832}

We present the case of a 13 year old girl who presented with spontaneous, recurrent and painful soft tissue swellings affecting her extremities. On several occasions the degree of swelling and pain was enough to consider compartment syndrome. To date she has required ten fasciotomies. On two occasions she has also had haematuria.

Baseline biochemical, haematological and radiological investigations were normal with no cause for symptoms identified. Skin biopsy showed no evidence of vasculitis. She underwent further extensive national investigations, including genetic testing for Type 4 Ehlers-Danlos syndrome. No pathological cause for purpura was found. Non-accidental and self inflicted injury were carefully considered, and excluded.

Following wide-ranging investigations and on review of her complex presentation she was diagnosed with Gardner-Diamond Syndrome (psychogenic purpura, autoerythrocyte sensitisation syndrome).

Gardner-Diamond Syndrome is a rare condition characterised by onset of spontaneous ecchymotic and painful lesions. The aetiology is not well understood but emotional stress is felt to be most common trigger for symptoms. Routine coagulation investigations are normal and the diagnosis is made clinically. It is therefore a diagnosis of exclusion.

This interesting case highlights a rare cause of painful purpura. A high index of suspicion was necessary to make the diagnosis. Numerous medical treatments have been trialled without any clear benefit. In this case, early administration of DDAVP has been beneficial in decreasing the progression of bruising, 
although the mechanism remains unclear. Other cases have also reported some improvement in symptoms following psychotherapy once the underlying cause of emotional stress has been identified.

\section{PO-0170 HEMOPHAGOCYTIC LYMPHOHISTIOCYTOSIS ASSOCIATED WITH VISCERAL LEISHMANIASIS: A CASE REPORT}

${ }^{1} \mathrm{~S}$ Ucar, ${ }^{2} \mathrm{P}$ Zorlu, ${ }^{3} \mathrm{NESE}$ Yarali, ${ }^{4} \mathrm{G}$ Tanir. ${ }^{1}$ Department of Pediatrics, OSM Middle East Hospital, Sanliurfa, Turkey; ${ }^{2}$ Department of Pediatrics, Dr. Sami Ulus Maternity and Children's Health and Diseases Training and Research Hospital, Ankara, Turkey; ${ }^{3}$ Department of Pediatric Hematology, Dr. Sami Ulus Maternity and Children's Health and Diseases Training and Research Hospital, Ankara, Turkey; ${ }^{4}$ Department of Pediatric Infection Diseases, Dr. Sami Ulus Maternity and Children's Health and Diseases Training and Research Hospital, Ankara, Turkey

\subsection{6/archdischild-2014-307384.833}

Background and aims Hemophagocytic lymphohistiocytosis $(\mathrm{HLH})$ is a rare, life threatening disorder characterizedby uncontrolled activation of T-helper 1 lymphocytes and macrophages and overproductionof inflammatory cytokines. Visceral leishmaniasis (VL), is a multisystem infection caused by the dissemination of Leishmania throughout the reticuloendothelial system. $\mathrm{HLH}$ associated VL is a rare and difficult to diagnose clinicopathological condition leading to delayed treatment. We report a rare case of HLH associated Visceral Leishmaniasis in a patient who presented with persistent high fever, hepatosplenomegaly and pancytopenia.

Case A 11 month-old baby with persistent high fever for more than 3 weeks and hepatosplenomegaly was assessed. Laboratory testing revealed pancytopenia, hypertriglyceridemia, hyperferritinemia and hypofibrinogenemia. Bone marrow examination revealed hemophagocytosis. A diagnosis of hemophagocytic syndrome was made according to the HLH-2009 diagnostic criteria. Prior to establishing the diagnosis of Leishmaniasis by indirect fluorescent antibody testing, several potential infectious agents were excluded. Treatment with pentavalent antimonials provided relief of all signs and symptoms within ten days.

Conclusion HLH should be included in the differential diagnosis of patients who present with persistent high fever, hepatosplenomegaly and pancytopenia. Besides, physicians should keep in mind Leishmaniasis among the potential etiologic factors of secondary HLH.

\section{P0-0171 FEATURES OF CLINICAL AND EPIDEMIOLOGICAL CHILDREN WITH SICKLE CELL ADMITTED IN TEACHING HOSPITAL ALCIDES CARNEIRO (HEAC), PETRÓPOLIS, RJ, BRAZIL}

E Veiga, A Veiga, A Ramos. Pediatria, Faculdade Medicina de Petrópolis, Petrópolis, Brazil

\subsection{6/archdischild-2014-307384.834}

Introduction According to the Ministry of Health, 3500 children are born each year with sickle cell disease in Brazil with $34.28 \%$ in Rio de Janeiro. Due to chronicity, complications and impact on the family and society, we choose to seek to know the evolving clinical features of sickle cell children seen in Petrópolis, Rio de Janeiro, Brazil.

Objective To describe the clinical and epidemiological characteristics of sickle cell anaemic children requiring hospitalisation in HEAC, Petrópolis, RJ, Brazil, in 2013.
Methodology Descriptive, retrospective, cross-sectional, quantitative study, documentary analysis of medical records of children hospitalised from January, 1 to December, 31, 2013, patients with sickle cell disease (SCD).

Results 11 patients were included and 12 hospitalizations. Diagnosis made by neonatal screening in 54.54\%. Males: $63.63 \%$. Mean age of 6.4 years $(12.4 \mathrm{y}-8 \mathrm{~m})$ Stay hospital ranged from 3 to 10 days (mean 6 days). All mentioned earlier hospitalizations. Nutritional assessment showed $81.8 \%$ of normal weight. Reasons for hospitalizations: $50 \%$ painful crises, pneumonia, $41.66 \%$ and $16.66 \%$ splenic sequestration. Need for blood transfusions in $50 \%$ of patients and $27.27 \%$ have splenectomized. Medical monitoring irregular in $36.47 \%$ patients.

Conclusion It is known that the prognosis of SCD is variable. Appropriate monitoring, workup appropriate therapeutic approach and the events should suit purposeful action strategies to reduce complications and also minimise the consequences resulting from the disease itself. Being the HEAC solely responsible for paediatric hospitalizations in the city, this study points to the implementation of protocols and control of action aimed at better prognosis of these children.

\section{P0-0172 BEHIND CLOSED DOORS: THE EFFECTS OF CHILDHOOD CANCER ON SOMATIC HEALTH OF PARENTS DURING AND AFTER ONCOLOGIC THERAPY}

${ }^{1} \mathrm{R}$ Wyrebek, ${ }^{1} \mathrm{G}$ Karpinsky, ${ }^{1} \mathrm{~B}$ Warszawski, ${ }^{1} \mathrm{P}$ Gorski, ${ }^{2} \mathrm{E}$ Bien, ${ }^{2} \mathrm{M}$ Krawczyk. ${ }^{1}$ English Division Pediatric Oncology Scientific Circle, Medical University of Gdansk, Gdansk, Poland; '2Department of Pediatrics Hematology and Oncology, Medical University of Gdansk, Gdansk, Poland

\subsection{6/archdischild-2014-307384.835}

Background Though surviving childhood cancer is a triumph for child and family, scars left on parents' health may linger long after treatment has been concluded.

Aim To analyse the impact of childhood cancer on somatic health of parents during treatment (DT: median 5.5 months) and after (AT: median 2 years).

Methods Analysis of 70 questionnaires (45 DT, 25 AT) from parents of cancer patients, treated at the Department of Paediatrics, Oncology and Haematology, Medical University of Gdansk, Poland, since 2011.

Results Over 60\% in both groups reported feeling physically worse since diagnosis and constantly worrying about their child. Parents DT had increased caffeine, nicotine and alcohol consumption, weight changes and hypertension. Two parents DT admitted to suicidal attempts, one developed cancer. Dermatological signs, mainly hair loss, were common both DT and AT. Both groups had sleeping difficulties; however, more AT experienced nightmares (20\% vs. $7 \%$ DT) and felt anxious and tired $(60 \%$ vs. $33 \%$ DT). Headaches, problems concentrating and migraines prevailed AT (60\%, 44\% and 28\% vs. 44\%, $29 \%$ and $7 \%$ DT; respectively). Cardiologic symptoms (tachycardia, dyspnea), gastrointestinal (gastroesophageal reflux, diarrhoea) and joint/bone pains predominated AT. $28 \%$ of parents AT felt unhappy with their intimate relationship (vs. 9\% DT). Only $20 \%$ of parents consulted with psychiatrist.

Conclusion Diagnosis of paediatric cancer leaves devastating somatic sequelae in parents, affecting all systems and life activities. Interestingly, parents AT report somatic complications more often than those DT. Medical assistance should be promoted for parents of cancer children even after termination of oncologic therapy. 